WINNY HIROME TAKAHASHI

Reginaldo Guedes Coelho Lopes $^{2}$

Daniella De Batista Depes ${ }^{3}$

Hosana Karine de Marathaoan SOUZa

Martins e Castello Branco ${ }^{4}$

\title{
Ablação histeroscópica do endométrio: resultados após seguimento clínico de 5 anos
}

\section{Results of bysteroscopic endometrial ablation after five-year follow-up}

Artigo Original

\begin{abstract}
Palavras-chave
Hemorragia uterina/terapia Hemorragia uterina/complicações Histeroscopia/métodos Resultado de tratamento

Keywords

Uterine hemorrhage/therapy Uterine hemorrhage/complications Hysteroscopy/methods Treatment outcome
\end{abstract}

\section{Resumo}

OBJETIVO: Avaliar os resultados clínicos, após seguimento mínimo de 5 anos, de pacientes com sangramento uterino anormal de etiologia benigna que realizaram ablação endometrial, analisando a taxa de sucesso do tratamento em relação ao método, compreendida como satisfação da paciente e melhora do sangramento uterino anormal, bem como complicações tardias, fatores associados e recorrência dos sintomas. MÉTODOS: Estudo transversal conduzido após período mínimo de 5 anos de cirurgia em pacientes submetidas ao procedimento entre 1999 e 2004. Foram analisados os seguintes dados: faixa etária quando da realização da cirurgia, complicações imediatas e tardias, e fatores associados. Foi utilizado o modelo de regressão logística com cálculo da respectiva Odds Ratio (OR) para se observarem as possíveis associações existentes entre a taxa de sucesso da cirurgia e as variáveis analisadas. RESULTADOS: Cento e quatorze pacientes foram submetidas à ablação endometrial no período de Março de 1999 a Abril de 2004. O tempo mediano de seguimento foi de 82 meses. $\bigcirc$ modelo de regressão logística permitiu a predição correta do sucesso da ablação endometrial em 80,6\%. A idade relacionou-se diretamente com o sucesso do procedimento $(\mathrm{OR}=1,2 ; \mathrm{p}=0,003)$ e a ligadura tubária pregressa mostrou relação inversa com o sucesso da ablação endometrial $(O R=0,3 ; p=0,049)$. Dentre as pacientes com falha terapêutica, 21 (72,4\%) foram tratadas com histerectomia. Em uma das pacientes submetidas à histerectomia foi confirmada a presença de hidro-hematossalpinge ao exame anatomopatológico, caracterizando a síndrome da ligadura tubária pós-ablação. CONCLUSÃO: A ablação endometrial tem se mostrado uma opção de tratamento vantajosa, mantendo altos índices de satisfação das pacientes, mesmo em seguimentos a longo prazo A idade quando da ablação endometrial influenciou no sucesso terapêutico e mais estudos são necessários para avaliar os fatores que poderão futuramente influenciar na indicação do procedimento em casos selecionados.

\section{Abstract}

PURPOSE: To evaluate the clinical outcomes after a minimum period of 5 years of follow-up of patients with abnormal uterine bleeding of benign etiology who underwent endometrial ablation, analyzing the success rate of treatment defined as patient satisfaction and improvement in uterine abnormal bleeding, as well as late complications and factors associated with recurrence of symptoms. METHODS: A cross-sectional survey was conducted after a minimum period of 5 years after surgery in patients who underwent the procedure between 1999 and 2004. We analyzed the following data: age at the time of surgery, immediate and late complications and associated factors. Logistic regression with Odds Ratio (OR) calculation was performed to evaluate possible associations between the success rate of surgery and the analyzed variables. RESULTS: A total of 114 patients underwent endometrial ablation between March 1999 and April 2004. The median follow-up was 82 months. The logistic regression model allowed the correct prediction of the success of endometrial ablation in $80.6 \%$ of cases. Age was directly related to the success of the procedure $(O R=1.2 ; p=0.003$ ) and previous tubal ligation showed a negative association with the success of endometrial ablation ( $O R=0.3 ; p=0.049)$. Among the patients with treatment failure, 21 (72.4\%) underwent hysterectomy. In one of the hysterectomy cases, hydro/hematosalpinx was confirmed by the anatomopathological exam, characterizing the postablation-tubal sterilization syndrome. CONCLUSION: Endometrial ablation has proven to be a worthwhile treatment option, maintaining high rates of patient satisfaction, even over long-term follow-up. The age at endometrial ablation influenced the therapeutic success. Further studies are needed to evaluate the factors that may influence the future indication for the procedure in selected cases.
Correspondência

Winny Hirome Takahashi Rua Pedro de Toledo $1.800-4^{\circ}$ andar -

Vila Clementino

CEP: $04029-000$

São Paulo (SP), Brasi

Recebido

$21 / 06 / 2011$
Traballho realizado no Serviço de Ginecologia e Obstetrícia do Hospital do Servidor Público Estadual "Francisco Morato de Oliveira" HSPE-FMO - São Paulo (SP), Brasil.

'Programa de Pós-graduação (Mestrado), Instituto de Assistência Médica ao Servidor Público Estadual - IAMSPE - São Paulo (SP), Brasil.

2 Serviço de Ginecologia e Obstetrícia, Hospital do Servidor Público Estadual "Francisco Morato de Oliveira" - HSPE-FMO - São Paulo (SP), Brasil.

${ }^{3}$ Setor de Endoscopia Ginecológica, Hospital do Servidor Público Estadual "Francisco Morato de Oliveira" - HSPE-FMO - São Paulo (SP), Brasil.

${ }^{4}$ Hospital do Servidor Público Estadual "Francisco Morato de Oliveira" - HSPE-FMO - São Paulo (SP), Brasil. 


\section{Introdução}

O sangramento uterino anormal é uma queixa comum nas mulheres em idade reprodutiva e sua abordagem é, muitas vezes, um desafio para o ginecologista. O tratamento clínico é a primeira opção, com resultados variáveis e eventualmente transitórios ${ }^{1-3}$. A histerectomia é a única terapêutica definitiva e uma das cirurgias mais realizadas em todo o mundo.

A ablação endometrial surgiu como método alternativo para as pacientes com sangramento uterino anormal de natureza benigna, no qual a histerectomia era anteriormente a única opção. A principal indicação da ablação endometrial é o sangramento uterino anormal de origem benigna em mulheres com prole constituída, que não respondem ao tratamento clínico ou apresentam contraindicações ao mesmo ${ }^{1}$. A curto e médio prazo, são evidentes seus benefícios em relação aos riscos e complicações da histerectomia, com menor tempo cirúrgico, menos morbidades cirúrgicas, menor tempo de hospitalização, retorno mais rápido às atividades diárias e alto índice de satisfação das pacientes ${ }^{4-6}$.

As técnicas de ablação endometrial são classificadas como histeroscópicas (de primeira geração) ou não histeroscópicas (de segunda geração). As histeroscópicas são realizadas sob visão direta e seus resultados dependem, em parte, da habilidade do cirurgião. Incluem ressecção endometrial com alça, eletrocoagulação com rollerball, combinação de ressecção com eletrocoagulação e destruição com o Nd:YAG laser. As técnicas de segunda geração são menos complexas, requerem uma menor curva de aprendizado, com resultados semelhantes aos métodos histeroscópicos, porém a um custo mais elevado ${ }^{1-3,7}$. As complicações intra e pós-operatórias precoces da ablação endometrial incluem perfuração uterina, sangramento, infecção pélvica, lesões de vísceras abdominais, embolia gasosa e síndromes de sobrecarga hídrica, sendo a maioria possível de ser evitada com boa técnica e treinamento adequado do cirurgiãa $0^{3,8}$.

A recorrência do sangramento uterino anormal ocorre em 10 a 20\% após 1 ano de seguimento, e a incidência de histerectomia chega a $35 \%$ em estudos com maior tempo de seguimento ${ }^{1,9}$.

Além disso, complicações a longo prazo têm recentemente sido relatadas. Após a ablação endometrial, o miométrio é exposto e, com a saída do meio distensor, as paredes uterinas se colabam, havendo uma tendência natural a se unirem num processo de cicatrização. Isso causa uma contratura que reduz a cavidade uterina a uma estrutura tubular com frequente obstrução da área cornual. O tecido endometrial remanescente ou regenerado, principalmente nas áreas cornuais e porção tubária intramural, pode sangrar e causar hematometra central, hematometra cornual, menstruação retrógrada ou retardar o diagnóstico de câncer endometrial. Em pacientes submetidas a ligadura tubária prévia, o sangramento retrógrado para a porção proximal da tuba pode resultar em distensão dolorosa, sendo conhecida como síndrome da ligadura tubária pós-ablação $0^{10-13}$.

Há relatos de incidência de $10 \%$ de hematometra cornual sintomático e síndrome de ligadura tubária pósablação $^{11}$. Não há dados na literatura brasileira sobre esses aspectos.

Há poucos estudos publicados até o momento que apresentam seguimento pós-ablação endometrial maior que 5 anos, com o sucesso sendo avaliado pela taxa de amenorreia e/ou satisfação da paciente com o procedimento. É importante que se conheçam quais os fatores que influenciam o sucesso do tratamento e a recorrência do sangramento uterino anormal, para que se planejem uma melhor terapia e a seleção de pacientes.

Assim, este estudo teve como objetivo avaliar os resultados clínicos, após seguimento mínimo de 5 anos, de pacientes com sangramento uterino anormal de etiologia benigna que realizaram ablação endometrial, analisando a taxa de sucesso do tratamento em relação ao método e os fatores que influenciaram nesses resultados, bem como a recorrência dos sintomas e as complicações tardias.

\section{Métodos}

Foi realizado estudo transversal e retrospectivo envolvendo pacientes do Setor de Endoscopia Ginecológica do Serviço de Ginecologia e Obstetrícia do Hospital do Servidor Público Estadual de São Paulo "Francisco Morato de Oliveira" (HSPE-FMO). Após aprovação pelo Comitê de Ética em Pesquisa dessa instituição, foi realizada a análise das características clínico-cirúrgicas registradas em prontuário.

Os critérios de inclusão foram: pacientes com sangramento uterino anormal, sem sucesso com o tratamento clínico, na pré-menopausa, com histeroscopia diagnóstica prévia, histerometria $\leq 12 \mathrm{~cm}$, biópsia endometrial sem atipias citológicas, submissão pregressa à ablação endometrial pela técnica de ressecção endometrial com alça combinada à eletrocoagulação com rollerball e seguimento ambulatorial mínimo de 5 anos.

$\mathrm{O}$ valor da histerometria foi considerado normal até $10 \mathrm{~cm}$ e pouco aumentado até $12 \mathrm{~cm}$. Todo tecido ressecado foi encaminhado para análise histológica, incluindo pólipos endometriais ou miomas submucosos eventualmente presentes à ablação endometrial. A intervenção era considerada bem sucedida quando a paciente permanecia em amenorreia, hipomenorreia ou eumenorreia e referia estar satisfeita com o procedimento. 
O grau de satisfação foi avaliado durante a última consulta de seguimento ambulatorial de rotina, quando era questionada e anotada em prontuário a satisfação da paciente com o método (satisfeita ou insatisfeita). A necessidade de histerectomia ou nova ablação endometrial foi considerada como falha terapêutica.

As análises foram realizadas utilizando-se o pacote estatístico Statistical Package for Social Sciences (SPSS), versão 16.0. A partir dos dados, foi construída uma tabela contendo características clínico-demográficas das pacientes, sendo os dados apresentados em forma de média \pm desvio padrão (DP) ou mediana (mín-máx), conforme a distribuição dos valores (normal ou não), bem como em porcentagens. A determinação da taxa de sucesso das pacientes após a cirurgia foi avaliada utilizando-se do teste de proporção simples com valores expressos em porcentagem. Foi utilizado o modelo de regressão logística, sendo a variável dependente (dicotômica) o sucesso da ablação, com cálculo da respectiva razão de chances $(O d d s$ Ratio - OR) para se observarem as possíveis associações existentes entre o sucesso da cirurgia (variável dependente), e as variáveis idade, paridade, tipo de parto, uso de análogo de $\mathrm{GnRH}$ no pré-operatório, histerometria normal, cavidade anormal (pólipo ou mioma) e presença de ligadura tubária prévia (variáveis independentes). A análise de correlação entre as variáveis analisadas com o intuito de reconhecer possíveis influências sobre o modelo foi feito por meio do cálculo do r de Pearson (bicaudal). O nível de significância foi estabelecido como $5 \%$.

\section{Resultados}

No período de Março de 1999 a Abril de 2004 foram realizadas 172 ablações endometriais em nosso serviço. Destas, 16 não preencheram os critérios de inclusão (9 foram submetidas à técnica de ablação com balão endotérmico, 6 eram pacientes na pós-menopausa e 1 paciente não apresentava com sangramento uterino anormal) e 42 pacientes perderam seguimento, restando 114 pacientes para o estudo. As pacientes foram acompanhadas por um período mediano de 82 meses (6-126 meses), ou até a realização de histerectomia ou nova ablação endometrial. A descrição das características clínico-demográficas das pacientes está representada na Tabela 1 .

Após o tratamento cirúrgico inicial, 68 pacientes $(59,6 \%)$ apresentaram amenorreia e $24(21,1 \%)$ hipomenorreia ou eumenorreia. Do total, 22 pacientes $(19,3 \%)$ não apresentaram melhora do sangramento uterino anormal. Destas, 17 foram submetidas à histerectomia e uma foi submetida à nova ablação do endométrio.

Não foram relatadas complicações intraoperatórias. No pós-operatório precoce, houve um caso de piometra, com necessidade de antibioticoterapia intravenosa. A paciente evoluiu bem, permanecendo em amenorreia e satisfeita com o tratamento. A médio prazo, ocorreram três casos de hematometra, sem resolução após dilatação cervical, sendo realizado histerectomia em duas pacientes.

Dentre as pacientes com falha terapêutica, 21 $(72,4 \%)$ foram tratadas com histerectomia, $6(20,7 \%)$ declararam-se insatisfeitas devido à persistência de sangramento uterino anormal e $2(6,9 \%)$ insatisfeitas devido a outros motivos, como dor pélvica cíclica e distensão abdominal, apesar de se apresentarem em amenorreia. Entre as indicações de histerectomia, 17 (81\%) foram resultantes da persistência do sangramento uterino anormal, $2(9,5 \%)$ devido à hematometra de repetição e $2(9,5 \%)$ decorrentes da presença de dor pélvica cíclica pós-ablação, sendo que em 1 delas foi confirmada a presença de hidro-hematossalpinge ao exame anatomopatológico. Apenas uma paciente foi submetida à nova ablação endometrial, permanecendo eumenorreica até a conclusão deste estudo. Das histerectomias, $61,9 \%$ foram realizadas nos primeiros 2 anos após a ablação endometrial.

A idade na época da ablação variou de 29 a 59 anos, com mediana de 45 (29-59) anos entre as pacientes com sucesso terapêutico e (34-50) anos para as que apresentaram insucesso, com diferença significativa entre os dois grupos $(\mathrm{p}=0,004)$.

A Tabela 2 mostra as variáveis analisadas e suas predições em relação à taxa de sucesso das pacientes

Tabela 1. Características clínico-demográficas de pacientes submetidas à ablação endometrial

\begin{tabular}{lc}
\hline Parâmetros & Média DP ou Mediana (mín-máx) ou n (\%) \\
\hline Idade (anos) & $44,5 \pm 5,5$ \\
Número de gestações & $2(0-16)$ \\
Número de partos & $2(0-12)$ \\
Parło vaginal & $1(0-11)$ \\
Parło cesária & $1(0-4)$ \\
Tempo de seguimento (meses) & $81(6-126)$ \\
Ligadura fubária prévia & $59(51,8)$ \\
Histerectomia & $21(18,4)$ \\
Complicações & $4(3,5)$ \\
Retrafamento & $22(19,3)$ \\
\hline
\end{tabular}

DP: desvio padrão; mín: mínimo; máx: maximo. 
Tabela 2. Análise das variáveis analisadas e suas predições em relação à taxa de sucesso das pacientes após ablação endometrial

\begin{tabular}{lccc}
\hline & OR & (IC95\%) & Valor $\mathrm{p}$ \\
\hline Idade & 1,2 & $1,1-1,3$ & 0,003 \\
Número de gestações & 0,7 & $0,4-1,4$ & 0,4 \\
Parto vaginal & 1,8 & $0,7-5,1$ & 0,2 \\
Parto cesária & 1,8 & $0,6-5,2$ & 0,2 \\
Ligadura tubária prévia & 0,3 & $0,06-0,9$ & 0,04 \\
Análogo de GnRH & 2,5 & $0,5-12,4$ & 0,2 \\
Histerometria normal & 0,6 & $0,1-2,2$ & 0,4 \\
Cavidade normal & 0,8 & $0,3-2,5$ & 0,7 \\
Complicações & 0,2 & $0,01-2,9$ & 0,2 \\
\hline
\end{tabular}

OR: Odds Ratio, IC95\%: intervalo de confiança de $95 \%$.

após ablação endometrial. Dentre as variáveis analisadas, a idade relacionou-se diretamente com o sucesso do procedimento $(\mathrm{OR}=1,2 ; \mathrm{p}=0,003)$ e a ligadura tubária pregressa mostrou relação inversa com o sucesso da ablação endometrial $(\mathrm{OR}=0,3 ; \mathrm{p}=0,049)$.

A análise da correlação entre as variáveis analisadas revelou correlação inversa entre uso de análogo de GnRH e idade $(r=-0,2 ; p=0,006)$, ou seja, quanto maior a idade, menor o uso de análogo de GnRH; correlação inversa entre o uso de análogo de GnRH e complicações pós-operatórias $(r=-0,2 ; \mathrm{p}=0,03)$; relação inversa entre complicações pós-operatórias e sucesso do procedimento $(\mathrm{r}=-0,2 ; \mathrm{p}=0,02)$; correlação direta entre ligadura tubária pregressa e complicações pósoperatórias $(r=0,2 ; p=0,05)$ e correlação direta entre idade e sucesso terapêutico $(r=0,2 ; p=0,007)$. Houve correlação entre ligadura tubária e paridade $(\mathrm{r}=0,5$; $\mathrm{p}<0,001)$.

Não foram encontradas correlações significativas entre o número de gestações, paridade, tipo de parto (vaginal ou cesariano), histerometria ou cavidade normal (ausência de mioma ou pólipo) e o sucesso terapêutico. De acordo com os resultados do exame anatomopatológico no pós-operatório, a presença de mioma, pólipo, adenomiose ou hiperplasia glandular simples não apresentou correlação significativa em relação ao sucesso após a cirurgia. Entre os achados do exame anatomopatológico resultantes do produto de histerectomia ou nova ablação endometrial, foram oito casos $(38,1 \%)$ de miomatose uterina, sete $(33,4 \%)$ de miomatose com adenomiose, dois $(9,5 \%)$ de adenomiose e quatro $(19 \%)$ sem outras particularidades.

Dentre as pacientes incluídas, 108 tiveram todos os parâmetros selecionados $(94,7 \%)$ e somente $6(5,3 \%)$ não tinham os dados completos. Após um seguimento clínico mínimo de 5 anos, a presença de ablações endometriais bem-sucedidas em 82 pacientes inicialmente permitiria uma predição de 75,9\% no modelo inicial,
Tabela 3. Resultados clínicos com o tratamento cirúrgico de ablação endometrial após seguimento ambulatorial mínimo de 5 anos

\begin{tabular}{|c|c|c|c|c|c|c|}
\hline \multicolumn{3}{|c|}{ Resultados clínicos } & $\begin{array}{c}\text { Sem } \\
\text { melhora }\end{array}$ & Amenorreia & $\begin{array}{c}\text { Hipo/ } \\
\text { eumenorreia }\end{array}$ & Total \\
\hline \multirow{4}{*}{ Sucesso } & \multirow{2}{*}{ Sim } & $\mathrm{n}$ & 0 & 63 & 22 & 85 \\
\hline & & $\%$ & 0,0 & 74,1 & 25,9 & 100,0 \\
\hline & \multirow{2}{*}{ Não } & $n$ & 22 & 5 & 2 & 29 \\
\hline & & $\%$ & 75,9 & 17,2 & 6,9 & 100,0 \\
\hline \multirow{2}{*}{ Total } & & $n$ & 22 & 68 & 24 & 114 \\
\hline & & $\%$ & 19,3 & 59,6 & 21,1 & 100,0 \\
\hline
\end{tabular}

dado que o número de ablações bem e mal-sucedidas diferem significativamente $(\mathrm{p}<0,001)$ (Tabela 3$)$. O modelo de regressão logística permitiu melhorar a predição correta do sucesso da ablação para $80,6 \%$. Dentre as mal sucedidas, obteve-se predição de $38,5 \%$ e, entre as bem-sucedidas, em 93,9\% dos casos.

\section{Discussão}

Em vários estudos publicados, a taxa de sucesso da ablação endometrial está em torno de 80 a $90 \%$, com risco de 15 a $35 \%$ das pacientes realizarem nova ablação endometrial ou histerectomia nos primeiros 5 anos $^{1,14-17}$. Já em série e com maior tempo de seguimento (mínimo de 5 anos) publicados até o momento, a taxa de sucesso da ablação endometrial tem variado de 67 a $92 \%{ }^{18-24}$. Fürst et al. ${ }^{18}$, em 10 anos de seguimento, encontraram satisfação de 84,3 (em uma escala analógica visual em que 0 era "insatisfeita" e 100 era muito satisfeita"), com $94 \%$ das pacientes recomendando o tratamento a sua melhor amiga e taxa de histerectomia de $22 \%$ ao final do período, sendo que $38 \%$ das histerectomias foram realizadas nos primeiros 2 anos $^{18}$. Comino e Torrejón ${ }^{20}$, em um período de 6 a 12 anos, realizaram $17,9 \%$ de histerectomias pós-ablação, com $81,2 \%$ das histerectomias sendo realizadas nos primeiros 2 anos. A taxa de sucesso foi de $81,8 \%$ após 5 anos de ablação endometrial, que manteve-se estável após 8 anos de acompanhamento $(80 \%)^{19}$. Em um período de seguimento mediano de 82 meses, nosso estudo encontrou grau de satisfação semelhante, de $80,6 \%$. Vinte e uma pacientes realizaram histerectomia e uma nova ablação endometrial, o que representa uma taxa de $19,3 \%$ de necessidade de nova abordagem cirúrgica, com $61,9 \%$ das histerectomias sendo realizadas nos primeiros 2 anos de seguimento.

Em relação à idade, apesar de alguns estudos discordantes ${ }^{15,20,24}$, mulheres com mais de 45 anos apresentaram melhores resultados cirúrgicos que mulheres mais jovens ${ }^{16,18,23,25}$. Assim, Fürst et al. ${ }^{18}$, após 10 anos de acompanhamento, evidenciaram taxa de histerectomia de 
$43 \%$ em pacientes com menos de 40 anos e de $18 \%$ nas com mais de 40 anos. Longinotti et al. ${ }^{23}$ também encontraram taxa de histerectomia de $12 \%$ em mulheres com mais de 50 anos, $19,8 \%$ entre 45 e 50 anos, $31 \%$ entre 40 e 45 anos e $40,6 \%$ em mulheres com menos de 40 anos $^{23}$. Do mesmo modo, também encontramos correlação direta significativa entre idade e sucesso terapêtico. À medida que aumenta a idade da paciente, também aumenta em $20 \%$ a chance de haver sucesso na cirurgia.

Em alguns estudos a médio prazo, a presença de ligadura tubária prévia mostrou-se fator de risco para histerectomia e dor pélvica pós-ablaçãa ${ }^{16,26}$. Em estudos de maior seguimento, tal fato não se repetiu ${ }^{15,20}$. Encontramos relação inversa entre a ligadura tubária pregressa e o sucesso da ablação endometrial, ou a ligadura tubária pregressa diminui o sucesso terapêutico em quase $70 \%$. Também encontramos correlação direta entre ligadura tubária pregressa e complicações pósoperatórias. Contudo, a síndrome da ligadura tubária pós-ablação (SLTPA) foi por nós comprovada em apenas uma paciente $(1,7 \%)$, do mesmo modo que em outra série, também com relato de um caso $(4,2 \%)^{15}$. Outros autores, no entanto, relatam taxas mais elevadas de SLTPA, com variação entre 6 e $8,4 \%$, respectivamente, todas confirmadas com exame anatomopatológico ${ }^{11,26,27}$ confirmadas com exame anatomopatológico ${ }^{26,27}$. El-Nashar et al. ${ }^{25}$, entre 816 mulheres submetidas à ablação endometrial, encontraram 9 pacientes que se apresentaram com dor pélvica e coleção intracavitária à ultrassonografia: em 3 foram evidenciadas hematometra e no restante não houve comprovação ao exame anatomopatológico. Como não foi solicitado ao patologista cortes seriados da área cornual uterina e segmento tubário proximal para confirmar o diagnóstico da SLTPA ou hematometra cornual, isso pode ter influenciado nossos resultados.

Entre as complicações no pós-operatório, tivemos três casos de hematometra $(2,4 \%)$ diagnosticados clinicamente e confirmados por meio da ultrassonografia. Destes, dois foram submetidos à histerectomia. Números semelhantes foram obtidos em seguimento de $200 \mathrm{pa}$ cientes, com 5 casos de hematometra $(2,5 \%)$, todos após 1 ano de ablação endometrial.
Assim relatado por outros autores ${ }^{18,23,25}$, a persistência do sangramento uterino anormal foi a principal indicação de histerectomia pós-ablação endometrial em nosso estudo, com $81 \%$ dos casos, seguido por dor pélvica ou associação de dor pélvica e sangramento uterino anormal.

A relação entre mioma uterino ou pólipo endometrial e o sucesso da ablação endometrial tem se mostrado na literatura de formas divergentes. Menor taxa de sucesso quando mioma ou pólipo estivessem presentes ${ }^{19,20}$ não evidenciou diferença em relação à presença de mioma ou pólipo e a taxa de histerectomia ou amenorreia ${ }^{16,23,25}$. Também não encontramos diferença significativa em relação ao sucesso do procedimento e a presença de mioma, pólipo, adenomiose ou hiperplasia glandular simples. Já de acordo com o exame anatomopatológico resultante do produto de histerectomia ou de nova ablação endometrial, tivemos predomínio de miomatose uterina seguido de adenomiose, assim como Carey et al. ${ }^{28}$. Viscomi et al..$^{21}$ encontraram adenomiose em todas as mulheres que foram submetidas à histerectomia após falha da ablação endometrial.

Apesar de serem complicações pouco frequentes, o hematometra e a SLTPA devem ser cogitados sempre que uma paciente se apresente com dor pélvica pós-ablação endometrial juntamente de amenorreia ou hipomenorreia. Também quando possível, deve-se solicitar ao patologista cortes seriados da área cornual uterina e segmento tubário proximal para confirmação diagnóstica.

A ablação endometrial tem se mostrado uma opção de tratamento vantajosa, mantendo altos índices de satisfação das pacientes, mesmo em seguimentos a longo prazo. A idade quando da ablação endometrial influenciou no sucesso terapêutico e a presença de ligadura tubária prévia sugere maior atenção às complicações no pós-operatório. $\mathrm{O}$ fato deste trabalho ter sido um estudo transversal de caráter histórico pode ter limitado a análise estatística e mais estudos são necessários para avaliar os fatores que poderão futuramente influenciar na indicação do procedimento em casos selecionados. Contudo, a ablação endometrial é método terapêutico valioso, que, sempre que possível, deve ser indicado às pacientes no tratamento de sangramento uterino anormal.

\section{Referências}

1. Garry R. Evidence and techniques in endometrial ablation: consensus. Gynaecol Endosc. 2002;11(1):5-17.

2. Lethaby A, Shepperd S, Cooke I, Farquhar C. Endometrial resection and ablation versus hysterectomy for heavy menstrual bleeding. Cochrane Database Syst Rev. 2004;(2):CD000329.
3. Crispi CP, Panisset KSP, Raymundo TS, Santos HCN, Rainho AFR, Azevedo MCL, et al. Ablação endometrial. In: Crispi CP, Oliveira FMM, Damian Junior JC, editores. Tratado de videoendoscopia e cirurgia minimamente invasiva em ginecologia. $2 a$ ed. Rio de Janeiro: Revinter; 2007. p. 1058-95. 
4. David SD, Depes DB, Ramos JFD, Lopes RGC, Lippi UG, Baracat FF, et al. Ressecção histeroscópica do endométrio: eficácia e fatores relacionados ao insucesso. Einstein. 2008;6(2): 120-4.

5. Rodrigues FF, Moura MS, Millen EC, Ramos JFD, Yatabe S, Lopes RGC, et al. Ressecção histeroscópica do endométrio: resultados e fatores de risco para insucesso. Rev Bras Ginecol Obstet. $2001 ; 23(7): 445-8$.

6. Dickersin K, Munro MG, Clark M, Langenberg P, Scherer R, Frick $\mathrm{K}$, et al. Hysterectomy compared with endometrial ablation for dysfunctional uterine bleeding: a randomized controlled trial. Obstet Gynecol. 2007; 1 10(6):1279-89.

7. Glasser $\mathrm{MH}$. Practical tips for office hysteroscopy and secondgeneration "global" endometrial ablation. J Minim Invasive Gynecol. 2009; 16(4):384-99

8. Scottish Hysteroscopy Audit Group. A Scottish audit of hysteroscopy surgery for menorrhagia: complications and follow-up. Br J Obstet Gynaecol. 1995;102(3):249-54.

9. Sharp HT. Endometrial ablation: what counts? Obstet Gynecol. 2010;1 16(4):808-9.

10. Townsend DE, McCausland VM, McCausland AM, Fields G, Kauffman K. Post-ablation -tubal sterilization syndrome. Obstet Gynecol. 1993;82(3):422-4.

11. McCausland AM, McCausland VM. Frequency of symptomatic cornual hematometra and postablation tubal sterilization syndrome after total rollerball endometrial ablation: a 10-year follow-up. Am J Obstet Gynecol. 2002;186(6):1274-80.

12. McCausland AM, McCausland VM. Long-term complications of endometrial ablation: cause, diagnosis, treatment, and prevention. J Minim Invasive Gynecol. 2007; 14(4):399-406.

13. Leung PL, Yuen PM. Postablation-tubal sterilization syndrome following thermal balloon endometrial ablation. Acta Obstet Gynecol Scand. 2006;85(4):504-5.

14. Martyn P, Allan B. Long-term follow-up of endometrial ablation. J Am Assoc Gynecol Laparosc. 1998;5(2):1 15-8.

15. Unger JB, Meeks GR. Hysterectomy after endometrial ablation. Am J Obstet Gynecol. 1996; 175(6): 1432-6.
16. Dutton C, Ackerson L, Phelps-Sandall B. Outcomes after rollerball endometrial ablation for menorrhagia. Obstet Gynecol. 2001;98(1):35-9.

17. Gandhi SV, Fear KBC, Sturdee DW. Endometrial resection: factors affecting long-term success. Gynaecol Endosc. 1999;8(1):41-50.

18. Fürst SN, Philipsen T, Joergensen JC. Ten-year follow-up of endometrial ablation. Acta Obstet Gynecol Scand. 2007;86(3):334-8.

19. Comino R, Torrejón R, Sánchez-Ortega I. Long-term results of endometrial ablation-resection. J Am Assoc Gynecol Laparosc. 2002;9(3):268-71

20. Comino R, Torrejón R. Hysterectomy after endometrial ablationresection. J Am Assoc Gynecol Laparosc. 2004; 11 (4):495-9.

21. Viscomi F, Martins JA, Pastore MD. Ablação histeroscópica do endométrio no tratamento da menorragia: seguimento de 200 casos. Rev Bras Ginecol Obstet. 2001;23(3):169-73.

22. Baggish MS, Sze EH. Endometrial ablation: a series of 568 patients treated over an 11 -year period. Am J Obstet Gynecol. 1996;174(3):908-13.

23. Longinotti MK, Jacobson GF, Hung YY, Learman LA. Probability of hysterectomy after endometrial ablation. Obstet Gynecol. 2008; $112(6): 1214-20$.

24. Panoskaltsis TA, Bassett PA, Frappell JM. Transcervical endometrial resection in 197 women. Hysterectomy rate and associated risk factors after a 5-8-year follow-up period. Gynaecol Endosc. 2002;11123):99-106.

25. El-Nashar S, Hopkins MR, Creedon DJ, Sauver JL, Weaver AL, $M c G r e e ~ M E$, et al. Prediction of treatment outcomes after global endometrial ablation. Obstet Gynecol. 2009;113(1):97-106.

26. Mall A, Shirk G, Van Voorhis BJ. Previous tubal ligation is a risk factor for hysterectomy after rollerball endometrial ablation. Obstet Gynecol. 2003;100(4):659-64.

27. Bae $I H$, Pagedas AC, Perkins HE, Bae DS. Postablation-tubal sterilization syndrome. J Am Assoc Gynecol Laparosc. 1996;3(3):435-8.

28. Carey ET, El-Nashar SA, Hopkins MR, Creedon DJ, Cliby WA, Famuyide AO. Pathologic characteristics of hysterectomy specimens in women undergoing hysterectomy after global endometrial ablation. J Minim Invasive Gynecol. 201 1;18(1):96-9. 